\title{
Endometriosis of the Inguinal Region, Case Report
}

\author{
Nagui Ross \\ Shouldice Hospital, Thronhill, Ontario, Canada \\ Email: rossna2@yahoo.com
}

Received 8 April 2014; revised 7 May 2014; accepted 6 June 2014

Copyright (C) 2014 by author and Scientific Research Publishing Inc.

This work is licensed under the Creative Commons Attribution International License (CC BY). http://creativecommons.org/licenses/by/4.0/

(c) (i) Open Access

\begin{abstract}
Endometriosis of the groin is a rare condition, with only 40 cases reported in literature. We here present 2 cases of this rare condition presented in the Shouldice Clinic, Thornhill, Ontario, Canada.
\end{abstract}

\section{Keywords}

\section{Endometriosis, Groin, Inguinal, Ultrasound}

\section{Introduction}

These two interesting cases highlight the necessity to include endometriosis in the differential diagnosis of lumpiness of the groin.

Endometriosis of the groin is a rare condition. Forty cases only have been identified in the literature; the first three were reported by Moloney in 1949 [1].

Two such patients presented themselves at the Shouldice Hospital in Thornhill, Ontario, Canada.

\section{Discussion}

The first case is a 47 years old female, who visited her family doctor complaining about lump in the left groin, after going into extensive investigations including 15 ultrasounds, one CAT scan, one MRI as well as one injection of thrombin under ultrasound guidance into that lesion for a preliminary diagnosis of pseudo aneurysm of the inferior epigastric artery.

Her surgical history consisted of an appendectomy in the 1970s and aspiration of the aforementioned false aneurysm of the inferior epigastric vessel. Her medical history was non-contributory she is married, has two children, is retired and does not smoke. She has allergies to penicillin and sulpha.

Upon physical examination there was a medium sized left inguinal region swelling measuring around $3 \times 5$ 
cm which was not completely reducible and gave an impulse on coughing.

Intra operative findings showed a $5 \times 10 \mathrm{~cm}$ multi-loculated hemorrhagic cyst which was loosely attached to the fundus of a medium sized indirect inguinal hernia sac. The cyst was carefully excised and sent to pathology; the indirect sac was freed and reduced into the abdominal cavity.

A pathology report revealed a fluid filled sac with marked reactive changes, focal endometriosis and no finding of malignancy (Figure 1, Figure 2). The patient was released from the hospital on the third post operative day; she was contacted two weeks later when she reported favorable post operative course and pain free recovery.

The second case is a 37 years old female with irreducible lump in the right groin with no impulse on coughing, however reporting tenderness and pain that increases during menstruation.

Upon exploration of the groin through a lower inguinal incision, that lesion was excised which was arising from the round ligament of the uterus, again the lump was sent to pathology where the results came as endometriosis of the round ligament. The patient was seen two weeks time later at which time she had had an uneventful

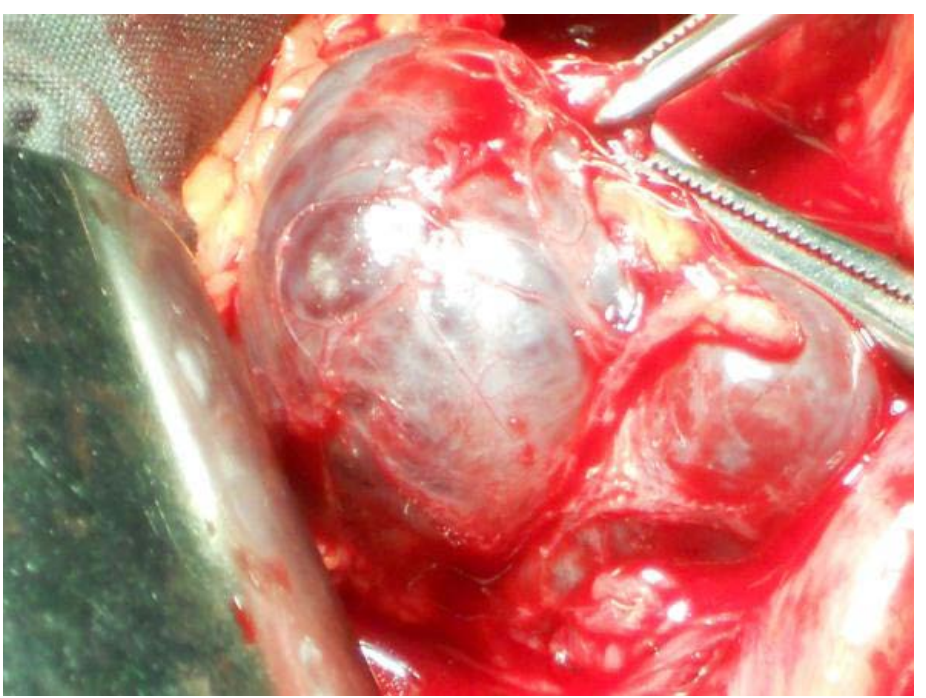

Figure 1. A photograph of the lesion in the first case prior to dissection from the adjacent hernia sac.

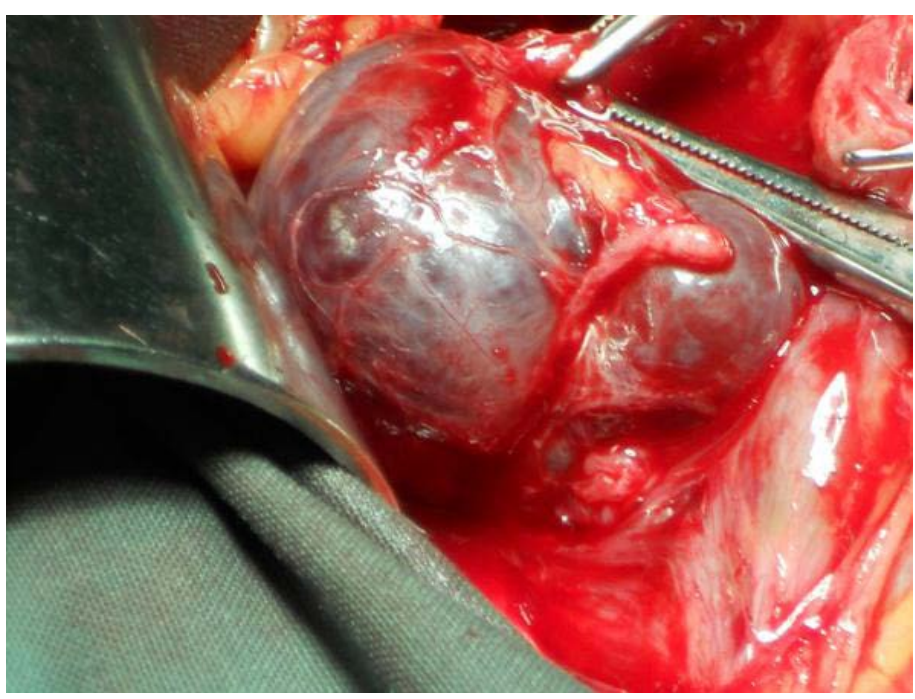

Figure 2. Shows the same lesion from another angle view depicting the color of the fluid inside the endometriotic cyst. 
recovery and was pain free.

These two interesting cases highlight the necessity to include endometriosis in the differential diagnosis of lump/mass in the groin in female patients [2].

Extensive and costly investigation may be curtailed by simple exploration of the groin to authenticate pathology and its probable association with inguinal hernias.

Although endometriosis is a common gynaecological diagnosis, inguinal endometriosis is a rare condition with $90 \%$ of the reported cases of extra-uterine endometrios is found in the right groin.

Curiously, our first reported case was left sided. Inguinal endometriosis is associated with inguinal hernia in approximately $32 \%$ of cases. It is advisable when the diagnosis has been made to consider the possibility of peritoneal seeding [3].

\section{Conclusion}

CT scanning and ultra sonography cannot provide an accurate diagnosis for these lesions so that a surgical exploration becomes a reasonable, recommended approach. The reason the overwhelming majority of cases of inguinal endometriosis being right sided is not known. A proposed theory has been that of a mechanically protective effect of the sigmoid colon which interferes with the coelomic metaplasia and with the dissemination of lymphatic and vascular migration and dissemination of these ectopic rests [4].

\section{References}

[1] Moloney, G.E. (1949) Endometriosis of the Groin; Report of Three Cases. British Medical Journal, 1, 435-437. http://dx.doi.org/10.1136/bmj.1.4601.435

[2] Clausen, I. and Nielson, K.T. (1987) Endometriosis in the Groin. International Journal of Gynecology Obstetrics, 25, 469-471. http://dx.doi.org/10.1016/0020-7292(87)90064-6

[3] Boereboom, C.L., Watson, N.F.S., Sivakumar, R., Atwal, G. and Tierney, G.M. (2009) Case Report: Endometriosis Presenting as an Acute Groin Swelling. Cases Journal, 2, 6438. http://dx.doi.org/10.4076/1757-1626-2-6438

[4] Majeski, J. (2001) Scar Endometriosis Manifested as a Recurrent Inguinal Hernia. Southern Medical Journal, 94, 247249. 
Scientific Research Publishing (SCIRP) is one of the largest Open Access journal publishers. It is currently publishing more than 200 open access, online, peer-reviewed journals covering a wide range of academic disciplines. SCIRP serves the worldwide academic communities and contributes to the progress and application of science with its publication.

Other selected journals from SCIRP are listed as below. Submit your manuscript to us via either submit@scirp.org or Online Submission Portal.
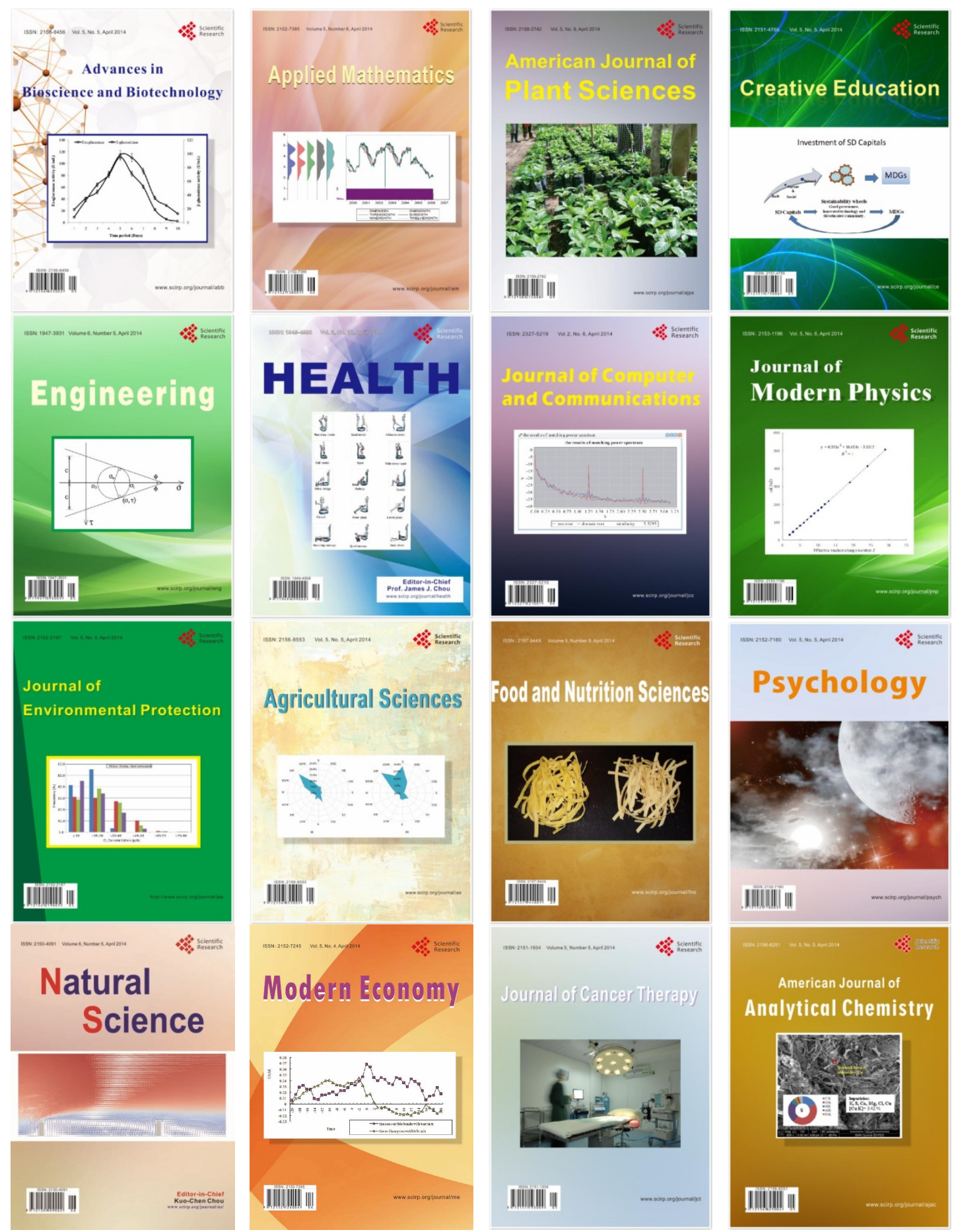